\title{
Identification of Cysteine-Rich Angiogenic Inducer 61 as a Potential Antifibrotic and Proangiogenic Mediator in Scleroderma
}

\author{
Pei-Suen Tsou, Dinesh Khanna, and Amr H. Sawalha
}

Objective. We previously identified CYR61 as a histone deacetylase 5 (HDAC-5)-repressed gene in systemic sclerosis (SSc; scleroderma) endothelial cells (ECs). When overexpressed, cysteine-rich angiogenic inducer 61 (CYR61) promoted angiogenesis in SSc ECs. This study was undertaken to examine the role of CYR-61 in fibrosis and determine the mechanisms involved in CYR-61-mediated angiogenesis in SSc.

Methods. Dermal ECs and fibroblasts were isolated from biopsy specimens from healthy subjects and patients with SSc. CYR-61 level was determined by quantitative polymerase chain reaction, Western blotting, and enzymelinked immunosorbent assay. CYR-61 was overexpressed using a CYR61 vector or knocked down using small interfering RNA, and functional and mechanistic studies were then conducted in fibroblasts and ECs.

Results. Lower CYR61 messenger RNA levels were observed in dermal fibroblasts and ECs from SSc patients than in those from healthy controls. In SSc fibroblasts, overexpression of CYR-61 led to significant reduction in the expression of profibrotic genes, including COL1A1 $(P=0.002)$ and ACTA2 $(P=0.04)$, and an increase in the expression of matrix-degrading genes, including MMP1 $(P=0.002)$ and MMP3 $(P=0.004)$, and proangiogenic VEGF $(P=0.03)$. The antifibrotic effect of CYR-61 was further demonstrated by delay in wound healing, inhibition of gel contraction, inactivation of the transforming growth factor $\beta$ pathway, and early superoxide production associated with senescence in SSc fibroblasts. In SSc ECs, overexpression of CYR-61 led to increased production of vascular endothelial cell growth factor. The proangiogenic effects of CYR-61 were mediated by signaling through $\alpha \mathrm{V} \beta 3$ receptors and downstream activation of AMP-activated protein kinase, AKT, and the endothelial cell nitric oxide synthase/ nitric oxide pathway system.

Conclusion. CYR-61, which is epigenetically regulated by HDAC-5, is a potent antifibrotic and proangiogenic mediator in SSc. Therapeutic intervention to promote CYR-61 activity or increase CYR-61 levels might be of benefit in SSc.

\section{INTRODUCTION}

Systemic sclerosis (SSc; scleroderma) is a connective tissue disease that is characterized by immune activation, vascular abnormalities, and progressive fibrosis in the skin and internal organs. It is a rare disease with a prevalence of 150-300 cases per million in Europe and the US (1). This disease is associated with significant morbidity and can lead to life-threatening complications, including pulmonary arterial hypertension, interstitial lung disease, and scleroderma renal

Dr. Tsou's work was supported by the Arthritis National Research Foundation and the Scleroderma Foundation. Dr. Khanna's work was supported by NIH grants K-24-AR-063120 and UM1-Al-110557 from the National Institute of Arthritis and Musculoskeletal and Skin Diseases (NIAMS) and the National Institute of Allergy and Infectious Diseases (NIAID), respectively. Dr. Sawalha's work was supported by the NIH (NIAID grants R01-Al-097134 and U19-Al-110502 and NIAMS grant R01-AR-070148). crisis. Although the etiology of SSc is still unclear, activation of immune cells and abnormalities of fibroblasts and endothelial cells (ECs) contribute to the pathogenesis of the disease (2). In addition, dysregulation of epigenetic mechanisms in SSc has been implicated in various cell types, and the use of epigenetic modifying drugs has been shown to be beneficial in cells as well as in animal models of SSc (2).

We recently showed that overexpression of the antiangiogenic histone deacetylase 5 (HDAC-5) in SSc ECs contributes to impaired angiogenesis by repressing proangio-

Pei-Suen Tsou, PhD, Dinesh Khanna, MD, Amr H. Sawalha, MD: University of Michigan, Ann Arbor.

No potential conflicts of interest relevant to this article were reported. Address correspondence to Amr H. Sawalha, MD, Division of Rheumatology, University of Michigan, 1150 West Medical Center Drive, 5520 MSRB1, SPC 5680, Ann Arbor, MI 48109. E-mail: asawalha@umich.edu.

Submitted for publication October 5, 2018; accepted in revised form March 12, 2019. 
genic factors (3). We took an unbiased approach to examine genome-wide changes in chromatin accessibility after HDAC-5 knockdown in ECs using an assay for transposase-accessible chromatin with sequencing. HDAC-5 knockdown led to increased chromatin accessibility, and through bioinformatics analyses and functional assays we identified novel HDAC- 5 target genes associated with impaired angiogenesis in SSc ECs, including CYR61.

Cysteine-rich angiogenic inducer 61 (CYR-61) is a member of the CCN protein family (CYR-61, connective tissue growth factor [CTGF], nephroblastoma-overexpressed gene, and Wnt-1-inducible signaling pathway proteins 1-3), which plays important roles in development, inflammation, tissue repair, and a broad range of pathologic processes including fibrosis and cancer (4). CYR-61 assumes its diverse functions by its ability to bind different combinations of co-receptors. For example, CYR-61 promotes EC proliferation and survival through binding to $\alpha \vee \beta 3(5,6)$, but enhances fibroblast adhesion and senescence through binding to $\alpha 6 \beta 1$ and heparan sulfate proteoglycans (HSPGs) $(7,8)$. Since this matricellular protein supports angiogenesis $(6,9)$ and possesses antifibrotic properties $(8,10)$, we hypothesize that CYR-61 promotes angiogenesis and inhibits fibrosis in SSc ECs and fibroblasts, respectively. In this study, we characterized the expression and function of CYR-61 in diffuse SSc dermal fibroblasts, and further dissected the mechanisms involved in the proangiogenic properties of CYR-61 in this disease.

\section{PATIENTS AND METHODS}

Patients and controls. Two 4-mm punch biopsy specimens from the distal forearm of healthy controls and patients with SSc were obtained for fibroblast and EC isolation. Plasma samples from the study participants were also collected to assess plasma CYR-61 protein levels. All patients met the American College of Rheumatology/European League Against Rheumatism criteria for the classification of SSc (11). This study was approved by the Institutional Review Board of the University of Michigan and all study participants signed a written informed consent. The demographic and clinical characteristics of the study participants are summarized in Table 1.

Cell culture. Dermal fibroblasts and ECs were isolated and characterized as previously described (12-14). After digestion, ECs were purified using a CD31 MicroBead Kit (Miltenyi Biotec) and grown in EBM-2 media with growth factors (Lonza). Fibroblasts were maintained in RPMI supplemented with 10\% fetal bovine serum (FBS). Cells between passage 3 and 6 were used in all experiments.

CYR-61 overexpression. Overexpression of CYR-61 in ECs was induced as previously described (3). ECs were trans-
Table 1. Characteristics of the SSc patients and healthy controls*

\begin{tabular}{lc}
\hline Age, mean \pm SD years & $\begin{array}{c}\text { SSc patients } \\
(\mathrm{n}=62)\end{array}$ \\
\hline Sex, female/male & $55.8 \pm 1.7$ \\
dcSSc & $50 / 12$ \\
Disease duration, mean \pm SD years & 41 \\
Modified Rodnan skin thickness score, & $8.9 \pm 1.4$ \\
$\quad$ mean \pm SD & $12.2 \pm 1.3$ \\
Raynaud's phenomenon & 56 \\
\hline Early disease (<5 years) & 37 \\
Digital ulcers & 12 \\
Telangiectasias & 27 \\
Gastrointestinal disease & 47 \\
Interstitial lung disease & 29 \\
Pulmonary arterial hypertension & 16 \\
Renal involvement & 3 \\
\hline
\end{tabular}

* In the healthy control group $(n=40)$, there were 31 women and 9 men, and the mean \pm SD age was $49.5 \pm 2.5$ years. Except where indicated otherwise, values are the number of subjects. SSc = systemic sclerosis; dcSSc = diffuse cutaneous SSc.

fected with $1.65 \mu \mathrm{g}$ of CYR61 (control vector pCMV6-XL4; OriGene) and Lipofectamine 2000 (3.3 $\mu$; Invitrogen) for 24 hours in T12.5 flasks. Five hours after transfection, culture media were changed to allow cells to grow in endothelial growth medium supplemented with bovine brain extract (Lonza). To overexpress CYR61 in dermal fibroblasts, $1 \mu \mathrm{g}$ of CYR61 and $1 \mu \mathrm{l}$ of Lipofectamine 2000 were used to transfect the cells in a 12-well plate for a total of 48 hours.

CYR-61 knockdown. CYR-61 expression was knocked down using CYR61 small interfering RNA (siRNA) (On-Target Plus siRNA; Dharmacon), while a nontargeting siRNA (Dharmacon) was used as a control. Fibroblasts isolated from healthy controls were transfected with $350 \mathrm{nM}$ of siRNA using TransITTKO transfection reagent (Mirus Bio) for 48 hours before messenger RNA (mRNA) was collected.

Western blotting. After obtaining cell lysates from cultured cells, proteins were separated by sodium dodecyl sulfate-polyacrylamide gel electrophoresis and electroblotted onto nitrocellulose membranes. Antibodies used included phosphotransforming growth factor $\beta$ receptor type II (phospho-TGF $\beta$ RII; Thermo Scientific), CYR-61, TGF $\beta R I I, p 21$, and p16 (all from Abcam), phospho-retinoblastoma protein (phospho-pRB), pRB, TGF $\beta$, phospho-Smad2, phospho-Smad3, Smad2/3, p53, phospho-p38, p38, phospho-endothelial cell nitric oxide synthase (phospho-eNOS), eNOS, phospho-AKT, AKT, phosphoAMP-activated protein kinase (phospho-AMPK), and AMPK (all from Cell Signaling Technology). $\beta$-actin was used as a loading 
control (Sigma-Aldrich). Band quantification was performed using Image J software (15).

\section{Messenger RNA extraction and quantitative reverse transcriptase-polymerase chain reaction (PCR). Total RNA from ECs and fibroblasts was isolated using a Direct-zol RNA MiniPrep kit (Zymo Research). A Verso complementary DNA (cDNA) synthesis kit was used to prepare cDNA (Thermo Sci- entific). Primers for human CYR61, COL1A1, ACTA2, PPARG, MMP1, MMP3, TGFB, TGFBR2, VEGF, FGF2, CDK1A, and $A C T B$ along with Power SYBR Green PCR Master Mix (Applied Biosystems), were used in quantitative PCR, which was run on a ViiA 7 Real-Time PCR System. Primers used were KiCqStart SYBR Green primers from Sigma-Aldrich or QuantiTect Primer Assays from Qiagen.}

Enzyme-linked immunosorbent assay (ELISA). After transfection with CYR61 or control vectors, cell culture media were changed to RPMI (for fibroblasts) or EBM-2 (for ECs) with 0.1\% FBS and cultured overnight. The levels of CYR-61, matrix metalloproteinase 1 (MMP-1), MMP-3, vascular endothelial growth factor (VEGF), and basic fibroblast growth factor in cell culture supernatants were measured using ELISA kits from R\&D Systems. Absorbance at $450 \mathrm{~nm}$ in each well was read using a microplate reader.

$\boldsymbol{\beta}$-galactosidase measurement. To examine the effect of CYR-61 on cell senescence, we measured $\beta$-galactosidase using a Senescence $\beta$-Galactosidase Staining Kit from Cell Signaling Technology.

Gel contraction and cell migration assays. The gel contraction assay was conducted as previously described (16). Fortyeight hours after CYR-61 overexpression or knockdown, dermal fibroblasts were suspended in culture media at $2 \times 10^{6} \mathrm{cell} / \mathrm{s} / \mathrm{ml}$. A Cell Contraction Assay kit (Cell Biolabs) was used for gel contraction. The area of the gel was analyzed using ImageJ software (15). To evaluate the effect of CYR-61 on cell migration, we performed cell migration assays using SSc fibroblasts transfected with control or CYR61 vectors. Transfected cells were grown to confluence and a wound gap was created. Culture media were replaced with RPMI with $0.1 \%$ FBS, and pictures were taken using an EVOS XL Core Cell Imaging System (Life Technologies) at 0 hours and 48 hours after wounding. The gap difference was quantified using ImageJ software (15).

Immunofluorescence staining. Cells grown in 8-well chambers were fixed in $4 \%$ formalin and blocked. They were then probed with anti-Ki-67 antibodies (Abcam) or anti-human p21 antibodies (Abcam). Alexa Fluor antibodies (Invitrogen) were subsequently used. The nuclei were stained using DAPI (Invitrogen). Ki-67-positive and p21-positive cells were counted using ImageJ software (15). To measure superoxide levels, dihydroethidium (DHE; Invitrogen) was used. After fixation, DHE $(10 \mu M)$ was added to CYR61-transfected or control-transfected fibroblasts for 15 minutes, and then stained with DAPI. Fluorescence was detected using an Olympus FV500 confocal microscope and photographs were taken at 400x.

Nitrite/nitrate measurement. To examine whether increased nitric oxide (NO) was present after CYR-61 overexpression, nitrite and nitrate levels in cell culture supernatant, both stable metabolites for NO, were measured using an OxiSelect In Vitro Nitric Oxide (Nitrite/Nitrate) Assay Kit from Cell Biolabs.

Matrigel tube formation assay. In our previous study, we showed that CYR-61 overexpression in SSc ECs resulted in increased tube formation (3). To examine whether this effect is mediated through $\alpha \vee \beta 3$ and its downstream pathways, we pretreated CYR-61-overexpressing SSc ECs with neutralizing antibodies to $\alpha \vee \beta 3(10 \mu \mathrm{g} / \mathrm{ml}$ ) or inhibitors for AMPK (compound c; $10 \mu \mathrm{M}$ ) or AKT (LY297002; $20 \mu M$ ) pathways before performing Matrigel tube formation assays. Treated SSC ECs were suspended in EBM-2 with $0.1 \% \mathrm{FBS}$ and plated in 8-well Lab-Tek chambers coated with growth factor-reduced Matrigel (BD Biosciences). Cells were fixed and stained after 6 hours of incubation. Quantitation of the tubes formed by ECs was performed using the Angiogenesis Analyzer function in Image J software (15). Pictures of each well were taken using an EVOS XL Core Cell Imaging System (Life Technologies).

Statistical analysis. Results are expressed as the mean \pm $S D$. To determine the differences between the groups, Student's $t$ test, paired $t$-test, or one-way analysis of variance was performed using GraphPad Prism software, version 6. $P$ values less than 0.05 were considered significant.

\section{RESULTS}

CYR-61 expression in SSc. Since CYR-61 is secreted and detected in blood, we first measured CYR-61 levels in plasma collected from healthy controls and SSc patients. We observed no differences in CYR-61 levels between healthy controls and SSc patients, including after stratifying patients into the categories diffuse cutaneous SSc (dcSSc) or limited cutaneous SSc (IcSSc) (Figure 1A). In dermal fibroblasts, there was significant down-regulation of CYR61 mRNA levels in both dcSSc and IcSSc fibroblasts compared to normal fibroblasts (Figure 1B). Protein levels of CYR-61 were measured in dcSSc fibroblasts and were found to be similar to those in control fibroblasts, as indicated by levels of secreted CYR-61 in supernatants or CYR61 protein levels in cell lysates (Figures $1 \mathrm{C}$ and D).

Antifibrotic properties of CYR-61 in SSc. To evaluate the effect of CYR-61 in dermal fibroblasts, we first 
A

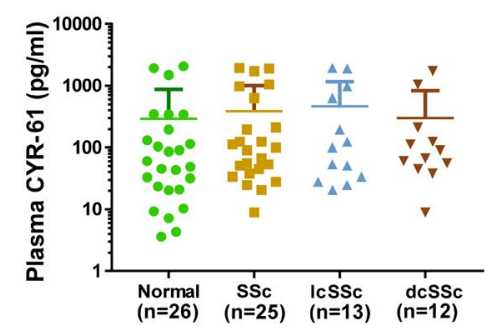

B

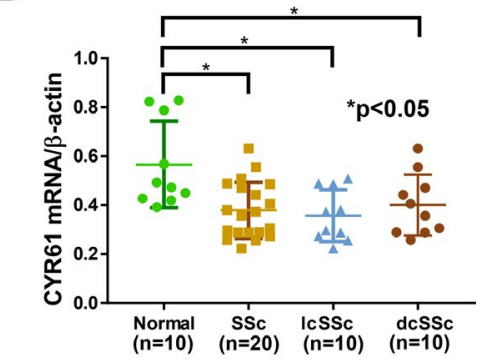

C

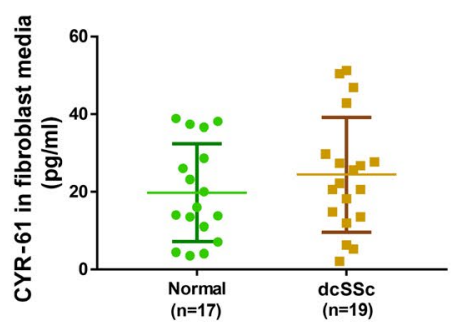

D

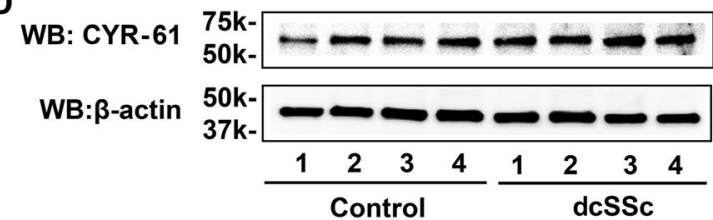

E

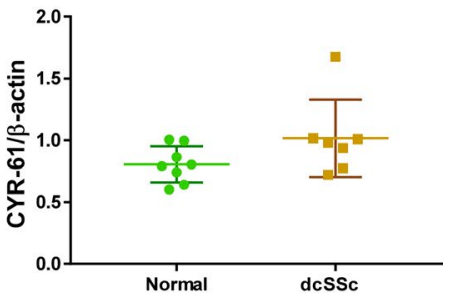

$\mathbf{F}$

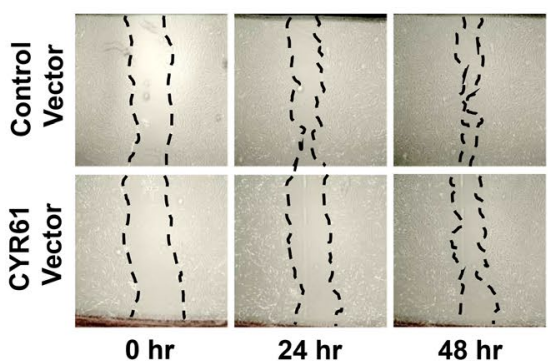

G
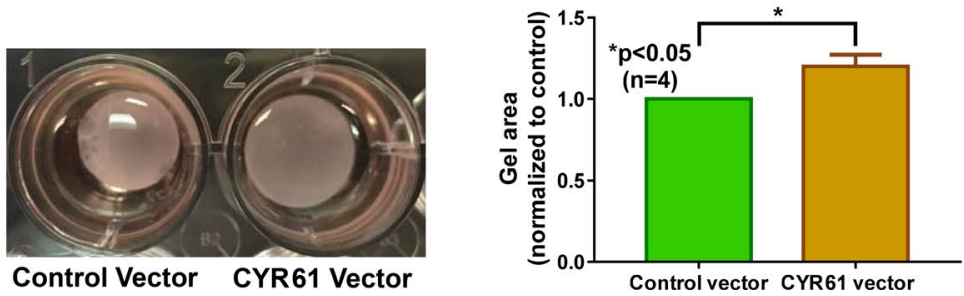

Figure 1. Antifibrotic role of cysteine-rich angiogenic inducer 61 (CYR-61) in dermal fibroblasts from patients with diffuse cutaneous systemic sclerosis (dcSSc). A, Expression of CYR-61 in plasma from healthy controls (normal), all SSc patients, patients with limited cutaneous SSc (ICSSc), and patients with dcSSc. There were no differences among the groups. B, Levels of CYR61 mRNA in healthy controls, all patients with SSc, patients with IcSSc, and patients with dcSSc. CYR61 mRNA expression was lower in both dcSSc and lcSSc dermal fibroblasts than in normal fibroblasts. C, CYR-61 levels in control and dcSSc fibroblast culture media. There was no difference between the 2 groups. D, Western blot (WB) (left) and Western blot band quantification (right) showing similar CYR-61 protein levels in normal and dcSSc dermal fibroblasts. E, Significant increases in CYR-61, matrix metalloproteinase 1 (MMP-1), MMP-3, and vascular endothelial growth factor (VEGF) in the culture media after overexpression of CYR-61 protein in dcSSc dermal fibroblasts. F, Illustration (left) and quantification (right) of wound area (in pixels) in SSc dermal fibroblasts after overexpression of CYR-61 using a CYR61 vector. At 48 hours the wound area was significantly wider in cells transfected with CYR61 vector than in cells transfected with control vector. G. Photograph (left) and quantification (right) of the gel area after overexpression of CYR-61 in dcSSc fibroblasts in a gel contraction assay. The gel area was increased in cells transfected with CYR61 vector. In A-D, symbols represent individual subjects; horizontal lines and error bars show the mean $\pm S D$. In G, bars show the mean \pm SD.

overexpressed CYR-61 in normal fibroblasts. Overexpression of CYR-61 led to a significant decrease in fibrotic markers, including COL1A1 and ACTA2, suggesting that CYR-61 is indeed antifibrotic in these cells (Supplementary Table 1, available on the Arthritis \& Rheumatology web site at http:// onlinelibrary.wiley.com/doi/10.1002/art.40890/abstract). We then overexpressed CYR-61 in dcSSc dermal fibroblasts. Similar to what was observed in normal fibroblasts, increased CYR-61 led to a significant reduction in COL1A1 and ACTA2 expression (Table 2). This was accompanied by a significant increase in the expression of antifibrotic PPARG as well as matrix-degrading MMP1 and MMP3. In addition to its effects on fibrosis-related genes, CYR-61 overexpression led to a significant increase in angiogenic factors, such as VEGF and FGF2, in dcSSc fibroblasts (Table 2).

At the protein level, overexpression of CYR-61 in dcSSc fibroblasts resulted in the release of significant amounts of CYR-61, MMP-1, MMP-3, and VEGF into the culture media (Figure 1E), suggesting that CYR-61 can act in both an autocrine and a paracrine manner. To further confirm the role of CYR-61 in dermal fibroblasts, we knocked down CYR-61 in normal fibroblasts and showed that down-regulation of CYR-61 led to a profibrotic phenotype, as indicated by elevated levels of COL1A1 and ACTA2 and decreased levels of MMP1 and MMP3 (Supplementary Table 2, available on the Arthritis \& Rheumatology 
Table 2. Expression levels of mRNA for selected target genes after CYR-61 overexpression in dcSSc dermal fibroblasts*

\begin{tabular}{lcc}
\hline Gene & $\begin{array}{c}\text { Fold change versus control- } \\
\text { transfected cells }\end{array}$ & $P$ \\
\hline CYR61 & $213 \pm 233$ & 0.002 \\
COL1A1 & $0.73 \pm 0.12$ & 0.002 \\
ACTA2 & $0.75 \pm 0.34$ & 0.04 \\
PPARG & $1.34 \pm 0.49$ & 0.01 \\
MMP1 & $3.70 \pm 5.32$ & 0.002 \\
MMP3 & $2.40 \pm 1.62$ & 0.004 \\
FGF2 & $1.42 \pm 0.53$ & 0.01 \\
VEGF & $1.45 \pm 0.98$ & 0.03 \\
\hline
\end{tabular}

* Values are the mean \pm SD fold change in expression in cells transfected with cysteine-rich angiogenic inducer 61 (CYR-61) versus control-transfected cells after transfection for 48 hours ( $n=10$ patients with diffuse cutaneous systemic sclerosis [dcSSc]).

web site at http://onlinelibrary.wiley.com/doi/10.1002/art.40890/ abstract).

To examine the antifibrotic effect of CYR-61 in SSc fibroblasts, we performed 2 functional assays: a scratch wound assay and a gel contraction assay. Overexpression of CYR-61 resulted in slower migration of dcSSc fibroblasts (Figure 1F) and reduced gel contraction (Figure 1G). In addition, CYR-61 knockdown in normal dermal fibroblasts resulted in increased gel contraction (Supplementary Figure 1, available on the Arthritis \& Rheumatology web site at http://onlinelibrary.wiley.com/ doi/10.1002/art.40890/abstract), providing further evidence of the antifibrotic properties of CYR-61 in SSc.

CYR-61 induces senescence in SSc fibroblasts. After confirming the antifibrotic functional role of CYR-61 in dcSSc fibroblasts, we examined the possible mechanism involved. We hypothesized that CYR-61-overexpressing SSc fibroblasts are converted from extracellular matrix (ECM)-producing myofibroblasts into ECM-degrading senescent cells, since CYR-61 has been shown to trigger senescence by increasing reactive oxygen species (ROS) and activating the p38 pathway through $\alpha 6 \beta 1$ integrin and HSPGs $(8,10,17)$. We first examined whether CYR-61 affects cell proliferation, using the proliferation marker $\mathrm{Ki}-67$. As shown in Figure 2A, dcSSc fibroblasts transfected with CYR61 showed a significant reduction in Ki-67 staining, indicating decreased proliferation. We then measured superoxide production after CYR-61 overexpression. We found that superoxide levels increased after 24 hours of CYR-61 overexpression, reaching a peak after 48 hours (Figure 2B). At 72 hours after transfection there was no difference in superoxide levels between control-transfected and CYR-61-transfected cells. These results suggest that superoxide production is an early event in the CYR-61-triggered senescence pathway. We then measured p21 expression, which is a marker for cell senescence. Both p21 mRNA and protein levels were signif- icantly elevated in CYR-61-overexpressing dcSSc fibroblasts (Figures 2C-E).

To further dissect the mechanism of CYR-61-mediated fibroblast senescence, we examined whether different senescence pathways were involved. Since we observed an increase in ROS production (Figure 2B), and ROS can activate stress-activated kinases such as MAPKs and downstream p38 MAPK (18), we first determined whether p38 MAPK was activated after CYR-61 overexpression in dcSSc dermal fibroblasts. Increased levels of phospho-p38 MAPK were indeed observed in CYR-61-overexpressing cells (Figure 2E). Because p38 MAPK activation results in increased transcriptional activity of p53 as well as up-regulation of p21 and p16, we further examined their expression in CYR-61overexpressing cells. Similar to phospho-p38 MAPK, increased accumulation of p53, p21, and p16 was also observed in fibroblasts transfected with the CYR61 vector compared to those transfected with control vector (Figure 2E). Since all triggers and signaling cascades involved in cell senescence converge on the hypophosphorylated form of pRB, we showed that overexpression of CYR-61 in dcSSc dermal fibroblasts led to a decrease in pRB phosphorylation (Figure 2E). Cell senescence after CYR-61 overexpression was also confirmed by $\beta$-galactosidase staining (Supplementary Figure 2, available on the Arthritis \& Rheumatology web site at http://onlinelibrary.wiley.com/doi/10.1002/ art.40890/abstract). These results indicate that the p38 MAPK, p53, and p16/phospho-pRB pathways contribute to CYR-61induced fibroblast senescence.

CYR-61 affects the TGF $\beta$ pathway in SSc fibroblasts. Because TGF $\beta$ is the most prominent growth factor in driving fibrosis and collagen production in SSc, we next investigated the effects of CYR-61 on TGF $\beta$ signaling in control-transfected and CYR61-transfected dcSSc dermal fibroblasts. We found that cells overexpressing CYR-61 showed attenuated phosphorylation of TGFBRII and Smad2/3 and reduced levels of TGF $\beta$. The expression levels of total TGFBRII and Smad2/3 did not change after CYR-61 overexpression (Figure 2E).

Characterization of proangiogenic mechanisms of CYR-61 in SSC ECs. We previously showed that CYR61 was overexpressed after HDAC-5 knockdown in ECs, and that it mediated the antiangiogenic effects of HDAC-5 in dcSSc ECs (3). To further confirm its role in SSc angiogenesis, we overexpressed CYR-61 in dcSSc ECs and showed that this led to increased angiogenic ability of SSc ECs (3). In the present study we further dissected the mechanisms involved in the proangiogenic properties of CYR-61 in SSc. We first determined whether CYR61 expression was altered in dcSSc ECs compared to normal ECs and found that CYR61 expression was lower in dcSSc ECs (Figure 3A). We hypothesized that overexpression of CYR-61 in SSc ECs leads to increased secretion of CYR-61 and other proangiogenic factors. We 
A

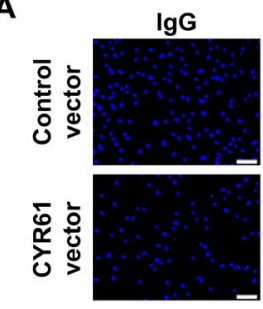

B

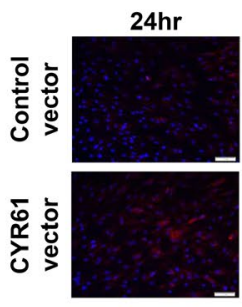

D

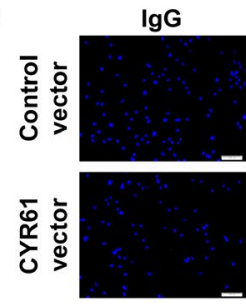

ki67

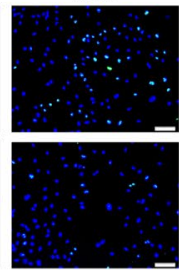

$48 \mathrm{hr}$

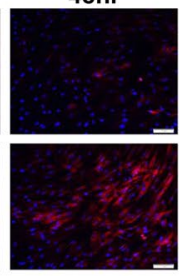

p21

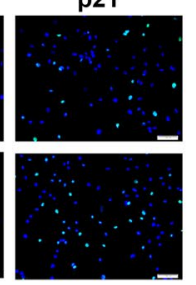

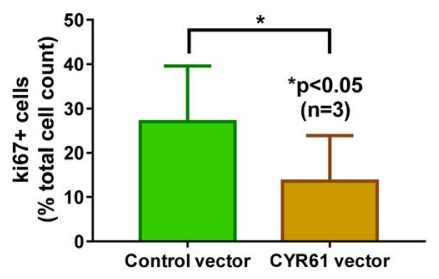

C
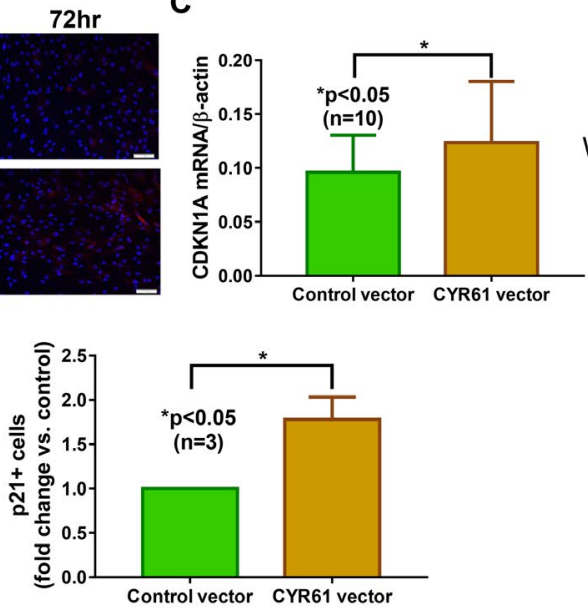

E

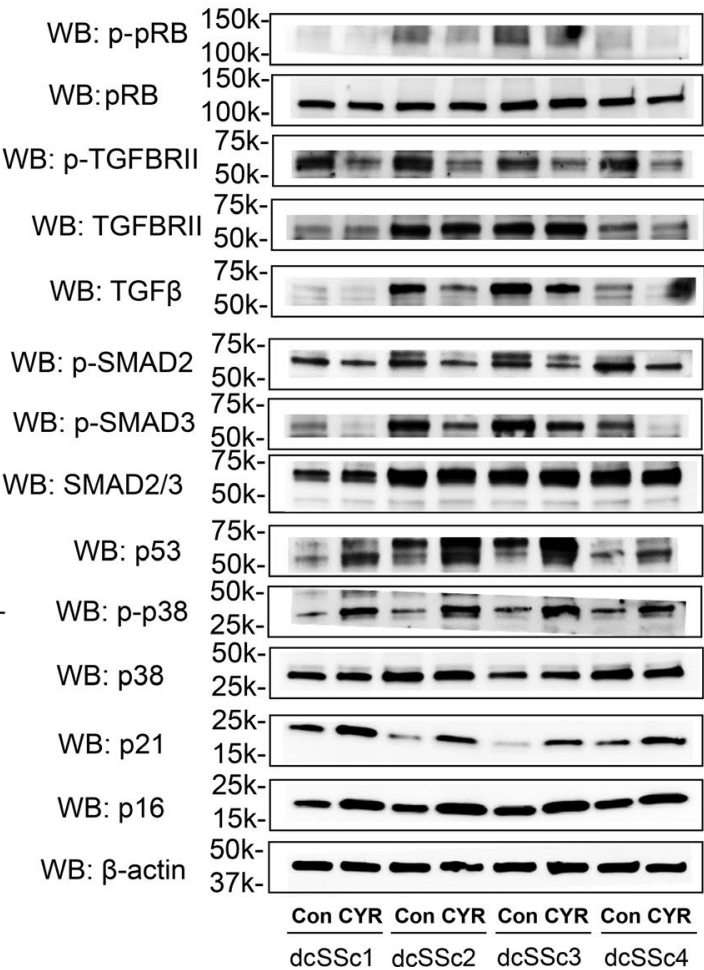

Figure 2. CYR-61-induced senescence in dcSSc dermal fibroblasts. A-D, Decreased cell proliferation after overexpression of CYR-61 in dcSSc dermal fibroblasts, as shown by Ki-67 staining (A), an increase in superoxide production that peaked at 48 hours (B), increased p21 (CDKN1A) mRNA levels (C), and increased p21 protein levels (D). In the left panels of $\mathbf{A}$ and $\mathbf{D}$ and in $\mathbf{B}$, scale bars $=10 \mu \mathrm{m}$. In the right panels of $\mathbf{A}$ and $\mathbf{D}$ and in $\mathbf{C}$, bars show the mean \pm SD. E, Western blot showing activation of senescence pathways and impairment of the transforming growth factor $\beta$ (TGF $\beta$ ) pathway in CYR-61-transfected dermal fibroblasts from 4 patients with dcSSc. The senescence pathways that were activated by CYR-61 in dcSSc dermal fibroblasts included p53, p38, p21, and p16, ultimately leading to hypophosphorylation of retinoblastoma protein (pRB). In addition, the antifibrotic effect of CYR-61 was mediated by inactivation of the TGF $\beta$ pathway, as indicated by reduced levels of phosphorylated TGF $\beta$ type II receptor and Smad2/3. See Figure 1 for other definitions.

focused on VEGF since it is reported to be a CYR-61-regulated gene (19). At the mRNA level, CYR-61 overexpression resulted in significant up-regulation of VEGF while FGF2, another proangiogenic mediator, remained unchanged (Figure 3B). We also measured secreted CYR-61 and VEGF in the culture media in control and CYR-61-overexpressing dcSSc ECs, and showed that overexpression of CYR-61 in dcSSc ECs increased both CYR-61 and VEGF secretion (Figures 3C and D).

To determine whether the receptor for CYR-61, $\alpha \vee \beta 3$, is present on ECs, we measured the mRNA levels in both normal and dcSSc ECs. There was a significant increase in ITGAV and ITGB3 expression in dcSSc ECs compared to normal cells (Figures $3 E$ and F). In contrast, ITGAV and ITGB3 levels did not change in CYR-61-overexpressing ECs (Supplementary Figure 3).

Since we established that the receptor for CYR-61, $\alpha \vee \beta 3$, is present on SSC ECs, and that overexpression of CYR-61 leads to increased CYR-61 and VEGF secretion, we then delineated the cellular events that were involved. CYR-61 was previously shown to promote angiogenesis in ECs by activating the AMPK pathway and the AKT/eNOS/NO pathway, both via $\alpha \vee \beta 3(20,21)$. Therefore, we first measured mRNA levels of
NOS3 (encoding eNOS) and found that it was up-regulated in CYR-61-overexpressing dcSSc ECs (Figure 3B). At the protein level the expression of eNOS was variable; however, when we measured phospho-eNOS, it was clear that CYR-61 overexpression led to eNOS activation (Figure 3G). To examine whether the eNOS-NO cascade is involved in CYR-61mediated angiogenesis, metabolites of $\mathrm{NO}$ were measured in culture media after control and CYR-61 overexpression. As expected, activation of eNOS led to a significant increase in NO metabolites in CYR-61-overexpressing cells (Figure 3H), suggesting that this pathway is involved in CYR-61-mediated angiogenesis in SSc ECs. We then examined whether the AMPK and AKT pathways were activated. As shown in Figure $3 G$, enhanced phosphorylation of AMPK and AKT were observed in CYR-61-overexpressing dcSSc ECs. Taken together, these results suggest that CYR-61 overexpression in dcSSc ECs leads to increased excretion of CYR-61, which, through binding to $\alpha \vee \beta 3$, activates the AMPK/AKT/eNOS pathways to promote angiogenesis.

To further confirm the involvement of $\alpha \vee \beta 3$ in CYR-61-mediated angiogenesis in SSc ECs, we performed Matrigel tube formation assays using CYR-61-overexpressing dcSSc ECs in 
A

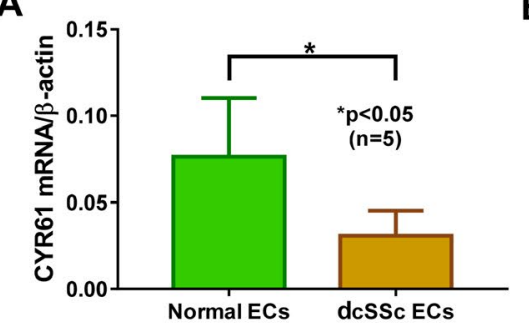

C

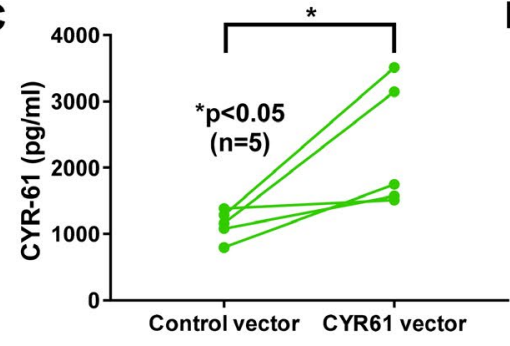

E

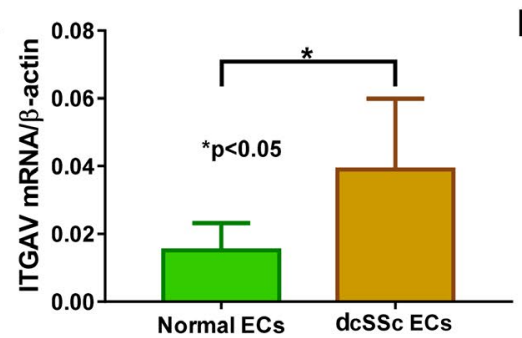

B

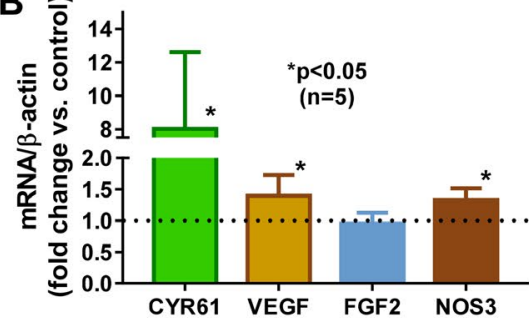

D

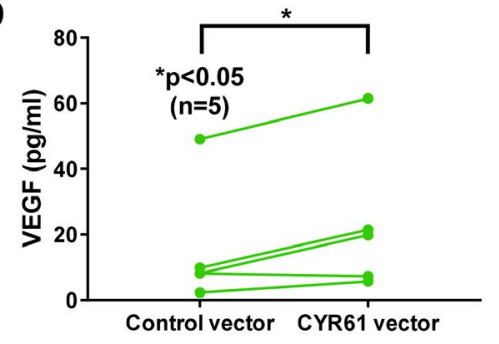

$\mathbf{F}$

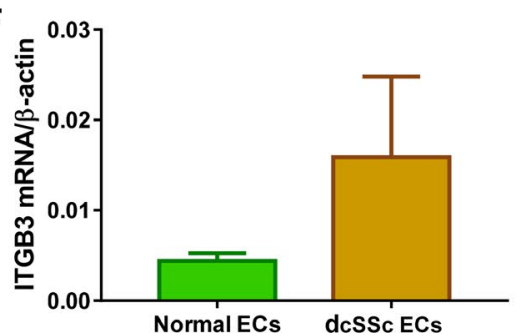

G

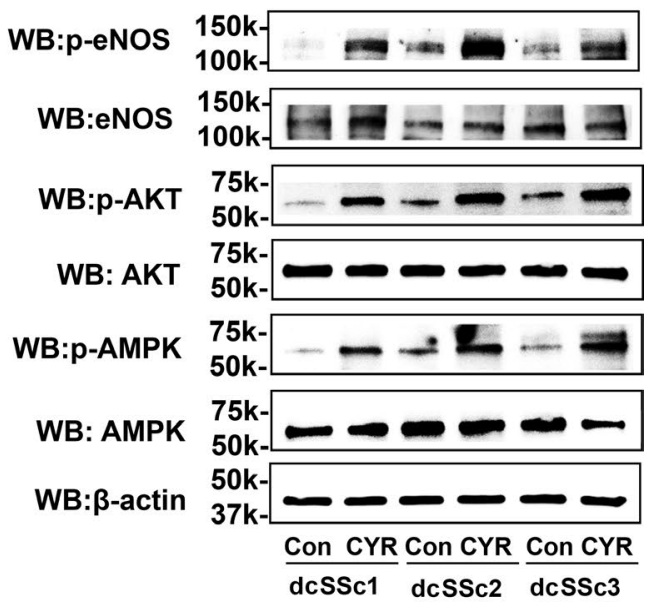

H

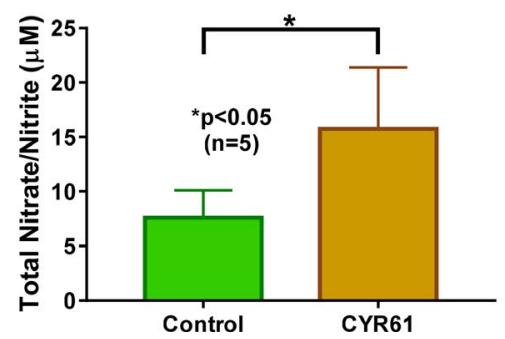

Figure 3. CYR-61 promotes angiogenesis in endothelial cells (ECs) from patients with dcSSc through $\alpha \vee \beta 3$ integrin, endothelial cell nitric oxide synthase (eNOS), AMP-activated protein kinase (AMPK), and AKT. A, Significantly lower CYR61 mRNA levels in dcSSc ECs than in normal ECs. B, Significant increases in CYR61, VEGF, and NOS3 after overexpression of CYR-61 in dcSSc ECs. C and D, Significant elevation of CYR-61 (C) and VEGF (D) levels in culture media from CYR-61-overexpressing dcSSc ECs. E and F, Elevated ITGAV (E) and ITGB3 (F) mRNA expression levels in dcSSc ECs compared to normal ECs. G, Western blot showing significant elevation of phosphorylated eNOS, AKT, and AMPK in CYR61-transfected dcSSc ECs. H, Increased levels of nitrates and nitrites (metabolites of nitric oxide) in culture media collected from CYR61-transfected dcSSc ECs. In A, B, E, F, and H, bars show the mean \pm SD. See Figure 1 for other definitions.

the presence or absence of blocking antibodies for $\alpha \vee \beta 3$. The proangiogenic property of CYR-61 was blocked by the presence of the $\alpha v \beta 3$ antibodies compared to the lgG isotype control (Figure 4A). In addition, inhibitors of AMPK (compound c) or AKT (LY294002) pathways blocked the proangiogenic activities of CYR-61 (Figure 4B).

\section{DISCUSSION}

In this study, we examined the effect of CYR-61 on both SSC fibroblasts and ECs. As illustrated in Figure 4C, overexpressing CYR-61 in SSc fibroblasts attenuates fibrogenesis by inducing cellular senescence and impairing the TGF $\beta$ pathway. We showed that overexpressing CYR-61 converted SSc fibroblasts from ECM-producing myofibroblasts into ECM-degrading senescent cells. This finding is supported by a reduction in fibrotic markers and an increase in matrix-degrading MMPs after overexpression of CYR-61 in SSc fibroblasts. In addition, the myofibroblast phenotype was inhibited, as shown by functional assays. We also demonstrated that markers for cell proliferation were reduced, key mediators for cell senescence were elevated, and the activation of TGF $\beta$ RII and downstream Smad pathways were inhibited. We believe this study provides sufficient evidence to support the notion that CYR-61 is indeed antifibrotic in SSc fibroblasts.

CYR-61 has been studied extensively in the skin, since it is a negative regulator of collagen homeostasis in fibroblasts, and is substantially elevated in aged and senescent human skin $(22,23)$. CYR-61 has been shown to trigger senescence by increasing ROS and activating the p38 pathway through $\alpha 6 \beta 1$ integrin and HSPGs $(8,10,17)$. In addition, it down-regulates TGF $\beta R \|$ and thereby impairs TGF $\beta$ responsiveness in fibroblasts $(17,24)$. Although CYR-61 blockade leads to improvement of fibrosis in lung and kidney fibrosis models $(25,26)$, CYR-61 has been shown to possess antifibrotic properties in animal models of cutaneous wound healing as well as liver fibrosis $(8,10,17)$. We were able to recapitulate the involvement of the p38 MAPK pathway in cell senescence in CYR-61-overexpressing SSc fibroblasts. However, instead of down-regulation of TGF $\beta R I I$, we showed decreased levels of TGF $\beta$, possibly leading to inactivation of TGF $\beta R$ II and its downstream Smad2/3 pathway in these cells. 
A
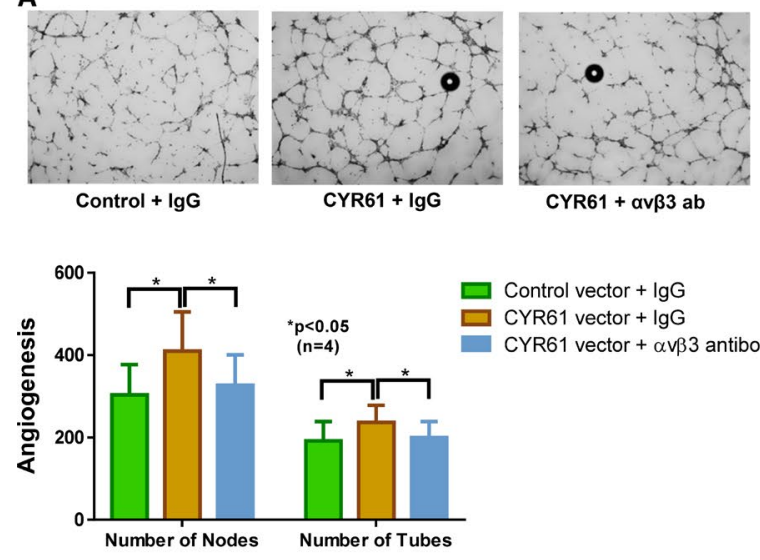

C

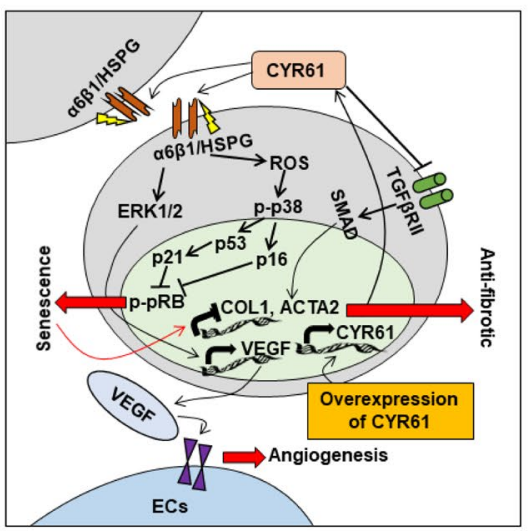

B
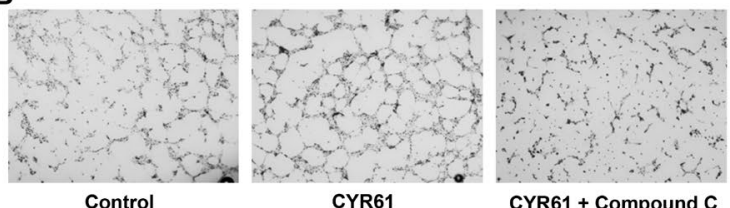

CYR61 + Compound C

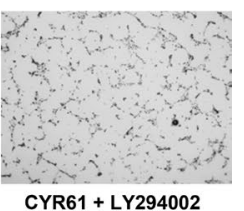

CYR61

CYR61 + LY294002

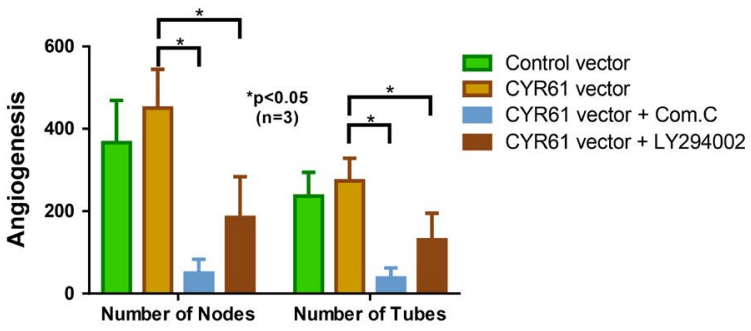

D

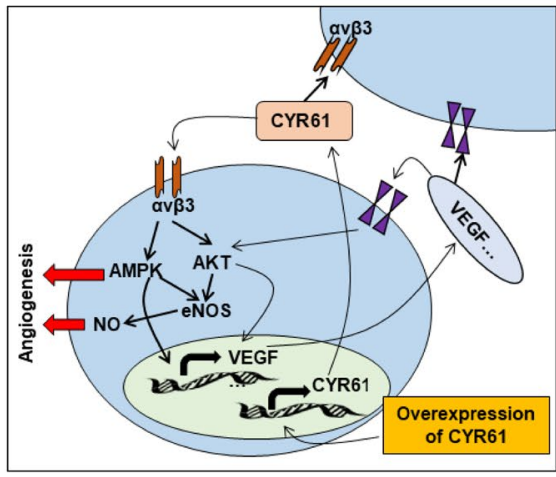

Figure 4. $\mathbf{A}$ and $\mathbf{B}$, Inhibition of the proangiogenic property of CYR-61 by blockade of $\alpha \vee \beta 3$ (A) or by inhibition of AMP-activated protein kinase (AMPK) or AKT (B). Photomicrographs (top panels) and quantification (bottom panels) of Matrigel tube formation assays using CYR61-overexpressing dcSSc endothelial cells (ECS) are shown. The proangiogenic activity of CYR-61 in promoting tube formation in dcSSc ECs was inhibited by $\alpha \vee \beta 3$ blocking antibodies (ab). Pretreating the dcSSc ECs with inhibitors for AMPK (compound c [com C]) or AKT (LY294002) reduced tube formation significantly after CYR-61 overexpression. Angiogenesis was shown by counting the number of nodes or the number of tubes, using ImageJ software. In top panels, original magnification $\times 4$; in bottom panels, bars show the mean \pm SD. C, CYR-61 overexpression in dcSSc fibroblasts. CYR-61 overexpression results in increased release of CYR-61 that binds to $\alpha 6 \beta 1$ integrin/heparan sulfate proteoglycan (HSPG). CYR-61 exerts antifibrotic properties by two mechanisms: 1) inducing senescence by reactive oxygen species (ROS) production, thereby activating p38/p16 or p38/p53/p21 pathways, and 2) attenuating the transforming growth factor $\beta$ (TGF $\beta$ ) pathway by inactivating the TGF $\beta / T G F \beta$ receptor type II (TGF $\beta$ RII) pathway. CYR-61 can also increase VEGF release from fibroblasts, which may increase the angiogenic potential of ECs. Studies have suggested that CYR-61 promotes VEGF production through activating the $\alpha 6 \beta 1$ integrin/HSPG-ERK pathway. D, CYR-61 overexpression in dcSSc ECs. CYR-61 overexpression results in increased CYR-61 excretion, leading to improved angiogenesis by binding $\alpha \vee \beta 3$ receptors and signaling through the AMPK/AKT/nitric oxide (NO) pathways. CYR-61 can also increase the expression and excretion of proangiogenic VEGF, possibly through the AKT and AMPK pathways. The proangiogenic effect of VEGF is known to be mediated through the AKT and its downstream NO signaling pathway. eNOS = endothelial cell nitric oxide synthase (see Figure 1 for other definitions).

Interestingly, another member of the CCN family, CCN2/ CTGF, is also involved in fibrosis. Although CYR-61 and CTGF share similar structures, receptors, and downstream signaling pathways, the subtle differences in their sequences alter the surface area and charge, thereby affecting their interactions with binding partners making them functionally unique (27). It is suggested that in wound healing, CYR-61 and CTGF act as yin and yang; CTGF promotes myofibroblast proliferation and matrix deposition to provide tissue integrity and promote wound repair when wounding occurs, while CYR-61 promotes wound resolution by inducing myofibroblast senescence and matrix degradation (8). CTGF, which promotes fibrosis, is elevated in
SSc patients and plays key roles in myofibroblast transformation through interaction with the TGF $\beta$ pathway (28-30). The imbalance between CTGF and CYR-61 could play a major role in promoting fibrosis in SSc.

It has been suggested that CYR-61 activates VEGF production in fibroblasts through the $\alpha 6 \beta 1 / \mathrm{HSPG} / \mathrm{ERK}$ axis (31). We indeed found that CYR-61-overexpressing SSc fibroblasts released elevated levels of VEGF (Figure 1E), prompting us to speculate that CYR-61 can promote EC angiogenesis through fibroblasts (Figure 4C). This is possible, since cross-talk between ECs and fibroblasts in SSc has been documented (32). Although VEGF has a limited effect on SSc ECs (the so-called VEGF par- 
adox), we showed previously that these cells do respond to higher levels of VEGF (33), which strengthens the argument that overexpressing CYR-61 in fibroblasts might increase the angiogenic potential of SSc ECs.

In addition to the effect of CYR-61 on fibroblasts, its effect on SSc ECs was also examined in this study (Figure 4D). CYR61 overexpression in SSc ECs led to increased excretion of CYR-61, which through binding to $\alpha v \beta 3$, activated the AMPK/ AKT pathways to promote angiogenesis. CYR-61 also activated expression of VEGF to further increase its proangiogenic potential. Both CYR-61 and proangiogenic factors can act in an autocrine or a paracrine manner. The involvement of $\alpha \vee \beta 3$ and the AKT and AMPK pathways in CYR-61-mediated angiogenesis was supported by the findings of our experiments with blocking antibodies and inhibitors. Our results are consistent with those of previous studies suggesting the involvement of the AMPK pathway and the AKT/eNOS/NO pathway in CYR61-mediated angiogenesis in ECs $(20,21)$. The ability of CYR61 to regulate VEGF was also documented (19). In addition, its angiogenic activity was observed in in vivo rat cornea models and ischemic rabbit hind limb models (20,21,34-38). Since CYR-61 is also critical for EC survival $(6,39)$ and increased apoptosis has been reported in SSc ECs (40), lower levels of CYR-61 in SSc ECs could contribute to both the impaired angiogenesis and the increased apoptosis seen in these cells.

Inconsistent results have been reported for CYR-61 serum levels in SSc $(41,42)$. Similar to what we observed, Saigusa et al (41) showed that serum CYR-61 levels were comparable among healthy controls, patients with dcSSc, and patients with IcSSc (41). They also showed that CYR-61 was downregulated in dermal small blood vessels of SSc patients compared to controls, which is consistent with what we observed in this study (41).

We previously showed that CYR-61 promotes EC tube formation in dcSSc ECs (3). In this study, we compiled evidence to indicate that CYR-61 is indeed proangiogenic and antifibrotic in SSc ECs and fibroblasts, respectively. Therefore, our data suggest that up-regulating CYR-61 in SSc might be of therapeutic potential in this disease. CYR-61 attenuated two key pathologic mechanisms in SSc: impaired angiogenesis and increased fibrosis. CYR-61 also modulates immune responses through macrophages $(43,44)$, and the possibility that CYR-61 inhibits macrophage-driven fibrosis by affecting macrophage polarization and monocyte/macrophage migration warrants further investigation.

\section{AUTHOR CONTRIBUTIONS}

All authors were involved in drafting the article or revising it critically for important intellectual content, and all authors approved the final version to be published. Dr. Sawalha had full access to all of the data in the study and takes responsibility for the integrity of the data and the accuracy of the data analysis.
Study conception and design. Tsou, Sawalha.

Acquisition of data. Tsou, Khanna.

Analysis and interpretation of data. Tsou, Sawalha.

\section{REFERENCES}

1. Barnes J, Mayes MD. Epidemiology of systemic sclerosis: incidence, prevalence, survival, risk factors, malignancy, and environmental triggers. Curr Opin Rheumatol 2012;24:165-70.

2. Tsou PS, Sawalha AH. Unfolding the pathogenesis of scleroderma through genomics and epigenomics. J Autoimmun 2017;83: 73-94.

3. Tsou PS, Wren JD, Amin MA, Schiopu E, Fox DA, Khanna D, et al. Histone deacetylase 5 is overexpressed in scleroderma endothelial cells and impairs angiogenesis via repression of proangiogenic factors. Arthritis Rheumatol 2016;68:2975-85.

4. Krupska I, Bruford EA, Chaqour B. Eyeing the Cyr61/CTGF/NOV (CCN) group of genes in development and diseases: highlights of their structural likenesses and functional dissimilarities. Hum Genomics 2015;9:24.

5. Grote K, Salguero G, Ballmaier M, Dangers M, Drexler H, Schieffer $\mathrm{B}$. The angiogenic factor $\mathrm{CCN} 1$ promotes adhesion and migration of circulating CD34+ progenitor cells: potential role in angiogenesis and endothelial regeneration. Blood 2007;110:877-85.

6. Leu SJ, Lam SC, Lau LF. Pro-angiogenic activities of CYR61 (CCN1) mediated through integrins $\alpha \vee \beta 3$ and $\alpha 6 \beta 1$ in human umbilical vein endothelial cells. J Biol Chem 2002;277:46248-55.

7. Chen CC, Young JL, Monzon Rl, Chen N, Todorović V, Lau LF. Cytotoxicity of TNF $\alpha$ is regulated by integrin-mediated matrix signaling. EMBO J 2007;26:1257-67.

8. Jun Jl, Lau LF. The matricellular protein CCN1 induces fibroblast senescence and restricts fibrosis in cutaneous wound healing. Nat Cell Biol 2010;12:676-85.

9. Kireeva ML, Mo FE, Yang GP, Lau LF. Cyr61, a product of a growth factor-inducible immediate-early gene, promotes cell proliferation, migration, and adhesion. Mol Cell Biol 1996;16:1326-34.

10. Kim KH, Chen CC, Monzon RI, Lau LF. Matricellular protein CCN1 promotes regression of liver fibrosis through induction of cellular senescence in hepatic myofibroblasts. Mol Cell Biol 2013;33: 2078-90

11. Van den Hoogen F, Khanna D, Fransen J, Johnson SR, Baron M, Tyndall A, et al. 2013 classification criteria for systemic sclerosis: an American College of Rheumatology/European League Against Rheumatism collaborative initiative. Arthritis Rheum 2013;65:273747.

12. Tsou PS, Amin MA, Campbell P, Zakhem G, Balogh B, Edhayan G, et al. Activation of the thromboxane A2 receptor by 8-isoprostane inhibits the pro-angiogenic effect of vascular endothelial growth factor in scleroderma. J Invest Dermatol 2015;135:3153-62.

13. Tsou PS, Rabquer BJ, Ohara RA, Stinson WA, Campbell PL, Amin MA, et al. Scleroderma dermal microvascular endothelial cells exhibit defective response to pro-angiogenic chemokines. Rheumatology (Oxford) 2016;55:745-54

14. He Y, Tsou PS, Khanna D, Sawalha AH. Methyl-CpG-binding protein 2 mediates antifibrotic effects in scleroderma fibroblasts. Ann Rheum Dis 2018;77:1208-18.

15. Schneider CA, Rasband WS, Eliceiri KW. NIH Image to ImageJ: 25 years of image analysis. Nat Methods 2012;9:671-5.

16. He Y, Tsou PS, Khanna D, Sawalha AH. Methyl-CpG-binding protein 2 mediates antifibrotic effects in scleroderma fibroblasts. Ann Rheum Dis 2018;77:1208-18.

17. Borkham-Kamphorst E, Schaffrath C, Van de Leur E, Haas U, Tihaa L, Meurer SK, et al. The anti-fibrotic effects of CCN1/CYR61 in primary portal myofibroblasts are mediated through induction 
of reactive oxygen species resulting in cellular senescence, apoptosis and attenuated TGF- $\beta$ signaling. Biochim Biophys Acta 2014;1843:902-14.

18. Muñoz-Espín D, Serrano M. Cellular senescence: from physiology to pathology. Nat Rev Mol Cell Biol 2014;15:482-96.

19. Mo FE, Muntean AG, Chen CC, Stolz DB, Watkins SC, Lau LF. CYR61 (CCN1) is essential for placental development and vascular integrity. Mol Cell Biol 2002;22:8709-20.

20. Park YS, Hwang S, Jin YM, Yu Y, Jung SA, Jung SC, et al. CCN1 secreted by tonsil-derived mesenchymal stem cells promotes endothelial cell angiogenesis via integrin $\alpha \vee \beta 3$ and AMPK. J Cell Physiol 2015;230:140-9.

21. Hwang S, Lee HJ, Kim G, Won KJ, Park YS, Jo I. CCN1 acutely increases nitric oxide production via integrin $\alpha \vee \beta 3$-Akt-S6Kphosphorylation of endothelial nitric oxide synthase at the serine 1177 signaling axis. Free Radic Biol Med 2015;89:229-40.

22. Quan T, Qin Z, Robichaud P, Voorhees JJ, Fisher GJ. CCN1 contributes to skin connective tissue aging by inducing ageassociated secretory phenotype in human skin dermal fibroblasts. $J$ Cell Commun Signal 2011;5:201-7.

23. Quan T, Qin Z, Voorhees JJ, Fisher GJ. Cysteine-rich protein 61 (CCN1) mediates replicative senescence-associated aberrant collagen homeostasis in human skin fibroblasts. J Cell Biochem 2012;113:3011-8

24. Quan T, He T, Shao Y, Lin L, Kang S, Voorhees JJ, et al. Elevated cysteine-rich 61 mediates aberrant collagen homeostasis in chronologically aged and photoaged human skin. Am J Pathol 2006;169:482-90.

25. Kurundkar AR, Kurundkar D, Rangarajan S, Locy ML, Zhou Y, Liu $\mathrm{RM}$, et al. The matricellular protein CCN1 enhances TGF- $\beta 1 /$ SMAD3dependent profibrotic signaling in fibroblasts and contributes to fibrogenic responses to lung injury. FASEB J 2016;30:2135-50.

26. Lai CF, Lin SL, Chiang WC, Chen YM, Wu VC, Young GH, et al. Blockade of cysteine-rich protein 61 attenuates renal inflammation and fibrosis after ischemic kidney injury. Am J Physiol Renal Physiol 2014;307:F581-92.

27. Holbourn KP, Acharya KR, Perbal B. The CCN family of proteins: structure-function relationships. Trends Biochem Sci 2008;33: 461-73.

28. Sato S, Nagaoka T, Hasegawa M, Tamatani T, Nakanishi T, Takigawa $M$, et al. Serum levels of connective tissue growth factor are elevated in patients with systemic sclerosis: association with extent of skin sclerosis and severity of pulmonary fibrosis. J Rheumatol 2000;27:149-54.

29. Holmes AM, Ponticos M, Shi-Wen X, Denton CP, Abraham DJ. Elevated CCN2 expression in scleroderma: a putative role for the TGF $\beta$ accessory receptors TGF $\beta R I I I$ and endoglin. J Cell Commun Signal 2011;5:173-7.

30. Abraham DJ, Shiwen X, Black CM, Sa S, Xu Y, Leask A. Tumor necrosis factor $\alpha$ suppresses the induction of connective tissue growth factor by transforming growth factor- $\beta$ in normal and scleroderma fibroblasts. J Biol Chem 2000;275:15220-5.
31. Chen CC, Mo FE, Lau LF. The angiogenic factor Cyr61 activates a genetic program for wound healing in human skin fibroblasts. J Biol Chem 2001;276:47329-37.

32. Serratì $S$, Chillà A, Laurenzana A, Margheri F, Giannoni E, Magnelli $\mathrm{L}$, et al. Systemic sclerosis endothelial cells recruit and activate dermal fibroblasts by induction of a connective tissue growth factor (CCN2)/transforming growth factor $\beta$-dependent mesenchymal-tomesenchymal transition. Arthritis Rheum 2013;65:258-69.

33. Tsou PS, Rabquer BJ, Ohara RA, Stinson WA, Campbell PL, Amin MA, et al. Scleroderma dermal microvascular endothelial cells exhibit defective response to pro-angiogenic chemokines. Rheumatology (Oxford) 2016;55:745-54.

34. Babic AM, Kireeva ML, Kolesnikova TV, Lau LF. CYR61, a product of a growth factor-inducible immediate early gene, promotes angiogenesis and tumor growth. Proc Natl Acad Sci U S A 1998;95:6355-60.

35. Fataccioli V, Abergel V, Wingertsmann L, Neuville P, Spitz E, Adnot S, et al. Stimulation of angiogenesis by Cyr61 gene: a new therapeutic candidate. Hum Gene Ther 2002;13:1461-70.

36. Hinkel R, Trenkwalder T, Petersen B, Husada W, Gesenhues F, Lee S, et al. MRTF-A controls vessel growth and maturation by increasing the expression of CCN1 and CCN2. Nat Commun 2014;5:3970.

37. Rayssac A, Neveu C, Pucelle M, Van den Berghe L, Prado-Lourenco $\mathrm{L}$, Arnal JF, et al. IRES-based vector coexpressing FGF2 and Cyr61 provides synergistic and safe therapeutics of lower limb ischemia. Mol Ther 2009;17:2010-9.

38. Chintala H, Krupska I, Yan L, Lau L, Grant M, Chaqour B. The matricellular protein $\mathrm{CCN} 1$ controls retinal angiogenesis by targeting VEGF, Src homology 2 domain phosphatase-1 and Notch signaling. Development 2015;142:2364-74.

39. Di Y, Zhang $Y$, Hui L, Yang $H$, Yang $Y$, Wang $A$, et al. Cysteinerich 61 RNA interference inhibits pathological angiogenesis via the phosphatidylinositol 3-kinase/Akt-vascular endothelial growth factor signaling pathway in endothelial cells. Mol Med Rep 2016;14:4321-7.

40. Wang $Y$, Kahaleh B. Epigenetic repression of bone morphogenetic protein receptor $\|$ expression in scleroderma. J Cell Mol Med 2013;17:1291-9.

41. Saigusa R, Asano Y, Taniguchi T, Yamashita T, Takahashi T, Ichimura $\mathrm{Y}$, et al. A possible contribution of endothelial CCN1 downregulation due to Fli1 deficiency to the development of digital ulcers in systemic sclerosis. Exp Dermatol 2015;24:127-32.

42. Lin J, Li N, Chen H, Liu C, Yang B, Ou Q. Serum Cyr61 is associated with clinical disease activity and inflammation in patients with systemic lupus erythematosus. Medicine (Baltimore) 2015;94:e834.

43. Bai T, Chen CC, Lau LF. Matricellular protein CCN1 activates a proinflammatory genetic program in murine macrophages. J Immunol 2010;184:3223-32.

44. Rother M, Krohn S, Kania G, Vanhoutte D, Eisenreich A, Wang X, et al. Matricellular signaling molecule CCN1 attenuates experimental autoimmune myocarditis by acting as a novel immune cell migration modulator. Circulation 2010;122:2688-98. 